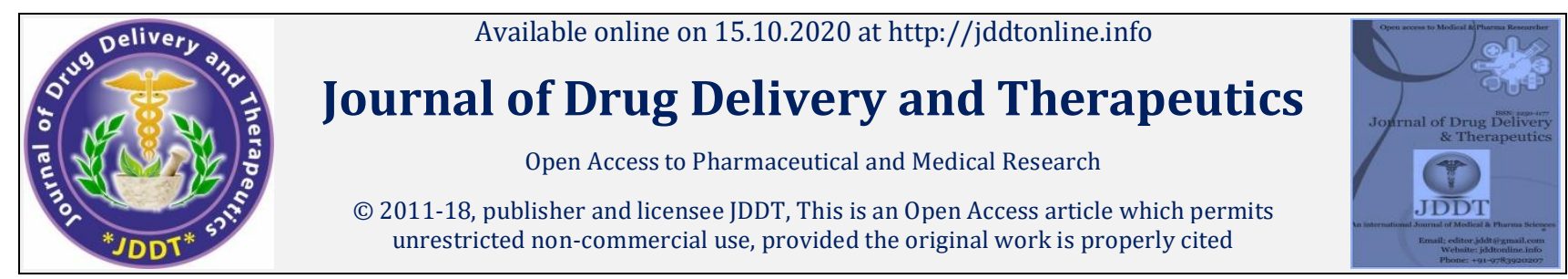

Open 8 Access

Review Article

\title{
A Review of an Ayurvedic Polyherbal Formulation Mustadi Kwatha
}

\author{
Hari Khanal ${ }^{*}$, Ram Kishor Joshi² ${ }^{2}$ Abhishek Upadhyay ${ }^{3}$ \\ 1 PG Scholar, Department of Kayachikitsa, National Institute of Ayurveda, Jaipur, Rajasthan, India \\ ${ }^{2}$ Professor and Head, Department of Kayachikitsa, National Institute of Ayurveda, Jaipur, Rajasthan, India \\ ${ }^{3}$ Lecturer, Department of Kayachikitsa, National Institute of Ayurveda, Jaipur, Rajasthan, India
}

\begin{abstract}
Mustadi Kwatha is a well-known Ayurvedic formulation. It is a widely prescribed drug and is used in all types of Prameha (Diabetes mellitus), Mutrakrichha (Urinary System related disease) and Santarpanjanya Vyadhi (disease due to over nutrition). Several studies have suggested that Mustadi Kwatha possesses anti-hyperlipidemic, anti- hyperglycemic and anti-oxidant activity. The present review explains the pharmacological potential of Mustadi Kwatha. In addition, this study investigates the pharmacological activities of each of the ingredients used in the formulation. It is believed that this review helps the researcher to further explore about this important Ayurvedic formulation.
\end{abstract}

Keywords: Mustadi Kwatha, Diabetes Mellitus, Hyperlipidemia, Obesity, Ayurvedic Polyherbal Formulation.

Article Info: Received 13 Aug 2020; Review Completed 18 Sep 2020; Accepted 27 Sep 2020; Available online 15 Oct 2020

Cite this article as:

Khanal H, Joshi RK, Upadhyay A, A Review of an Ayurvedic Polyherbal Formulation Mustadi Kwatha, Journal of Drug Delivery and Therapeutics. 2020; $10(5-\mathrm{s}): 267-272 \quad$ http://dx.doi.org/10.22270/jddt.v10i5-s.4448

*Address for Correspondence:

Dr. Hari Khanal, PG Scholar, Department of Kayachikitsa, National Institute of Ayurveda, Jaipur-302002, Rajasthan, India.

\section{INTRODUCTION}

Mustadi Kwatha is a polyherbal formulation widely used in many disorders due to its various pharmacological activities. It is one of the commonly used Ayurvedic formulation. It was mentioned in Ayurvedic classics in Charaka Samhita and in Bhaisajya Ratnavali. Acharya Charaka has stated that regular administration of Mustadi Kwatha can cure all types of Santarparjanya Vyadhi (disease due to over nutrition). ${ }^{1}$ In Bhaisajya Ratnavali it was mentioned that regular intake of $40 \mathrm{ml}$ of Mustadi Kwatha cures all type of Prameha (Diabetes mellitus) and Mutrkriiccha (Urinary System related disease). ${ }^{2}$ According to Acharya Charaka, Mustadi Kwatha consists of 13 medicinal herbs. However, as mentioned in Bhaisajya Ratnavali, this polyherbal drug consists of 9 herbs [Table 1]. Both type of formulation is mainly used in metabolic syndrome related disease. An extensive review of the ancient traditional literature and modern research revealed that the drug has numerous therapeutic actions like anti-hyperlipidemic, anti- hyperglycemic and antioxidant etc., several of which have been established scientifically, which may help the researchers to set their minds for approaching the utility, efficacy and potency of Mustadi Kwatha. A standard dose of Mustadi Kwatha is 40 to $50 \mathrm{ml}$ twice a day before meals.

\section{METHOD OF PREPARATION OF KWATHA}

Mixture of Yavakutta (coarse) powder of all dry herbs is to be taken in amount of 25 grams. After adding 16 times of water to coarse powder, it should be allowed for boiling in an open mouthed container on low fire. Boiling should be continued till it reduces to one-eighth. Then after filtering, it can be given to patient in lukewarm form. Each time fresh Kwatha should be prepared ${ }^{3}$

\section{AYURVEDIC PROPERTIES}

As per the description available in Ayurveda classics, therapeutic effect of medicine depends upon certain pharmacodynamics properties of its individual ingredients. These pharmacodynamics properties are Rasa, Guna, Veerya, Vipaka, Prabhava etc. Mustadi Kwatha therapeutic effect depend upon its individual herbs pharmacodynamics properties. [Table 3] 
Table 1: Ingredients of Mustadi Kwatha 4,5

\begin{tabular}{|l|l|}
\hline According to Acharya Charaka & According to Bhaisajya Ratnavali \\
\hline Nagarmotha (Cyperus rotundus Linn.) & Nagarmotha (Cyperus rotundus Linn.) \\
\hline Haritaki (Terminalia chebula Retz.) & Haritaki (Terminalia chebula Retz.) \\
\hline Amalaki (Emblica officinalis Gaertn.) & Amalaki (Emblica officinalis Gaertn.) \\
\hline Vibhitaki (Terminalia bellirica Roxb.) & Vibhitaki (Terminalia bellirica Roxb.) \\
\hline Haldi ((Curcuma longa Linn.) & Haldi (Curcuma longa Linn.) \\
\hline Devdaru (Cedrus deodara Loud.) & Devdaru (Cedrus deodara Loud.) \\
\hline Gokhuru (Tribulus terrestris Linn.) & Murva (Marsdenia tenacissima Wight.) \\
\hline Khadir (Acacia catechu Willd) & Lodhra (Symplocos racemosa Roxb.) \\
\hline Neem (Azadirachta indica A. Juss) & Indravaruni (Citrullus colocynthis Schrad.) \\
\hline Amaltas (Cassia fistula Linn.) & \\
\hline Daruharidra ( Berberis aristata DC) & \\
\hline Vatsaka (Holarrhena antidysenterica Linn.) & \\
\hline Patha (Cissampelos pareira Linn) & \\
\hline
\end{tabular}

\section{CHEMICAL COMPOSITION WITH PHARMACOLOGICAL ACTIVITIES}

Table 2: Ingredients of Mustadi Kwatha with their parts used, chemical composition and pharmacological activities.

\begin{tabular}{|c|c|c|c|}
\hline $\begin{array}{l}\text { Name of } \\
\text { the drug }\end{array}$ & Parts Used & Chemical Composition 6,7 & Pharmacological Activities \\
\hline Nagarmotha & Rhizome & $\begin{array}{l}\text { The major constituents include cineol(+) } \\
\text { copadiene, copaene, cyperen I\&II, cyperenone, } \\
\text { isopatchoulenone, cyperotundone, cyperol, } \\
\text { cyperolone, Beta-cyperone, kobusone, } \\
\text { mustakone, patchulene and } \beta \text { - sitosterol. }\end{array}$ & $\begin{array}{l}\text { Nagarmotha has anti-diabetic, anti-diarrheal, } \\
\text { anti-hyperlipidemic, hepatoprotective, anti- } \\
\text { bacterial, anti-inflammatory and anti-oxidant } \\
\text { action. } 8 \text { It also has diuretic, carminative, } \\
\text { anthelminthic, analgesic, and antirheumatic } \\
\text { activities. }^{9}\end{array}$ \\
\hline Haritaki & Fruit & $\begin{array}{l}\text { It contains anthraquinone glycoside, } \\
\text { chebulinic acid, chebulagic acid, tannic acid, } \\
\text { terchebin, tetrachebulin, vitamin } C \text {, linoleic etc. }\end{array}$ & $\begin{array}{l}\text { The main pharmacological activities of Haritaki } \\
\text { are Antioxidant, Anticarcinogenic, } \\
\text { Antimutagenic, radioprotective, } \\
\text { Chemopreventive, Hepatoprotective, } \\
\text { Cardioprotective, Antidiabetic, Antibacterial, } \\
\text { Antifungal, Antiviral, Antiprotozoal, Anti- } \\
\text { inflammatory, anti-arthritic activity, Anti- } \\
\text { allergic, Hypolipidemic, hypocholesterolemic, } \\
\text { Gastrointestinal motility improving, anti- } \\
\text { ulcerogenic, Wound healing and } \\
\text { Immunomodulatory.10 }\end{array}$ \\
\hline Bibhitaki & Fruit & $\begin{array}{l}\text { The major constituents include fructose, } \\
\text { galactose, glucose, Chebulagic acid, ellagic acid, } \\
\text { gallic acid, mannitol, rhamnose, } \beta \text { - sitosterol, } \\
\text { bellericanin and tannins. }\end{array}$ & $\begin{array}{l}\text { It has analgesic, antioxidant, hepatoprotective, } \\
\text { antibacterial, anticancer and immune- } \\
\text { modulatory activities. }{ }^{11}\end{array}$ \\
\hline Amalaki & Fruit & $\begin{array}{l}\text { The main chemical composition of Amalaki are } \\
\text { carotene, nicotinic acid, riboflavine, D-glucose, } \\
\text { D-fructose, myoinositol and a pectin with D- } \\
\text { galacturonic acid, D-arabinosyl, D-xylosyl, L- } \\
\text { rhamnosyl, D-glucosyl, D-mannosyl and D- } \\
\text { galactosyl residues, prodelphinidin tannins, } \\
\text { polyphenolic compounds; 1,2,3,6- } \\
\text { trigalloylglucose, terchebin, corialgin, ellagic } \\
\text { acid, alkaloids, phyllantidine and phyllantine. }\end{array}$ & $\begin{array}{l}\text { It has Anti-ageing, Antidiabetic, Anti } \\
\text { hyperthyroid, Anticancer, Antioxidant, } \\
\text { Nephroprotective, Hypolipidemic, } \\
\text { Immunomodulator, Hepato protective, } \\
\text { anticarcinogenic, Cardio protective action. }{ }^{12}\end{array}$ \\
\hline Haridra & Rhizome & $\begin{array}{l}\text { The major constituents include curcuminoids, } \\
\text { desmethoxycurcumin, bidesmethoxy } \\
\text { curcumin, phytosterols, fatty acids and } \\
\text { polysaccharides. }\end{array}$ & $\begin{array}{l}\text { Haridra has anti-diabetic, hepatoprotective, } \\
\text { cardioprotective, anti-inflammatory, anti- } \\
\text { bacterial, hypolipidemic, antioxidant, } \\
\text { antimicrobial and anti-carcinogenic activity. }{ }^{13}\end{array}$ \\
\hline Devdaru & Heartwood & $\begin{array}{l}\text { It contains Essential oil from wood; } \\
\text { Sesquiterpene, p-methylacetophenone, }\end{array}$ & $\begin{array}{l}\text { Cedrus deodara has anti-diabetic, anti-ulcer, } \\
\text { anti-inflammatory, anti-arthritic, anti- }\end{array}$ \\
\hline
\end{tabular}




\begin{tabular}{|c|c|c|c|}
\hline & & $\begin{array}{l}\text { atlantone. Steam bark contain deodarin and } \\
\text { toxifolin. }\end{array}$ & $\begin{array}{l}\text { convulsant, anxiolytic, anti-microbial, anti- } \\
\text { inflammatory, anti-viral, anti-tubercular, } \\
\text { immunomodulatory, antioxidant and wound } \\
\text { healing activity. }{ }^{14}\end{array}$ \\
\hline Murva & Root & $\begin{array}{l}\text { The major constituents include Pregnane } \\
\text { glycoside, tenacissoides, Marsdenin, D- } \\
\text { Cymarose, Asclepobiose, D-Canarose and } \\
\text { Cissogenin. }\end{array}$ & $\begin{array}{l}\text { It hasanti- diabetic, anti- inflammatory, } \\
\text { immunomodulatory, antioxidant, anti- tumor, } \\
\text { anti-pyretic, anti-carcinogenic activity. }{ }^{15}\end{array}$ \\
\hline Indravaruni & Root & $\begin{array}{l}\text { It contains mainly alkaloids I, II \& III; choline } \\
\text { cucurbitacin B, cucurbitains I \& II, citrullonol, } \\
\text { citrulluin, citrulluene, citrolic acid, elateridine, } \\
\text { hexanorcucubitacin I, cucurbitacin E, citrullol, } \\
\text { citronellal, methyleugenol, inositol etc. }\end{array}$ & $\begin{array}{l}\text { Indravaruni has Hypolipidemic, hypoglycemic, } \\
\text { anti-inflammatory, antioxidant and free radical } \\
\text { scavenging activity. }{ }^{16}\end{array}$ \\
\hline Lodhra & Bark & $\begin{array}{l}\text { The major constituents is Loturine } 0.24 \% \text {, } \\
\text { Colloturine } 0.02 \% \text {, Loturidine } 0.06 \% \text {. It also } \\
\text { contain symposide, epifzelechin, oxalic acid, } \\
\text { phytosterol, oleanolic and ellagic acids. }\end{array}$ & $\begin{array}{l}\text { It has hypolipidemic, anti-androgenic, anti- } \\
\text { diabetic, anti-inflammatory, anti-oxidant, anti- } \\
\text { ulcer, anti-angiogenic and hepatoprotective } \\
\text { activity. }{ }^{17}\end{array}$ \\
\hline Amaltas & $\begin{array}{l}\text { Pulp of pods, } \\
\text { Root Bark, } \\
\text { Leaf and } \\
\text { Flower }\end{array}$ & $\begin{array}{l}\text { The major constituents include } \\
\text { anthraquinones, flavonoids, flavon-3-ol } \\
\text { derivatives, alkaloid, glycosides, tannin, } \\
\text { saponin and terpenoids. }\end{array}$ & $\begin{array}{l}\text { Amaltas has anti-inflammatory, antioxidant, } \\
\text { antidiabetic, hypolipidemic, hepato-protective, } \\
\text { antimicrobial, antitumor, antiulcer, antipyretics, } \\
\text { analgesic and laxative activities. }{ }^{18}\end{array}$ \\
\hline Patha & $\begin{array}{l}\text { Root, } \\
\text { Rhizome }\end{array}$ & $\begin{array}{l}\text { It contains mainly Pelosine, Bebeerines, } \\
\text { Cycleanine, Saponine. Deyamittin, Cissamine, } \\
\text { isochondrodendrine, l-curine, menismine,, } \\
\text { pareirine, hayatinine, bisbenzylisoquinoline } \\
\text { and dicentrine. }\end{array}$ & $\begin{array}{l}\text { It has diuretic, antidiabetic, antiasthamatic, } \\
\text { hepatoprotective, anti-inflammatory, } \\
\text { antioxidant, antipyretic activities. }{ }^{19}\end{array}$ \\
\hline Gokharu & $\begin{array}{l}\text { Fruit and } \\
\text { Root }\end{array}$ & $\begin{array}{l}\text { The major constituents include Harman, } \\
\text { Harmine, glycosides, tannin,Volatail oil, } \\
\text { flavonoids, flavonol, steroidal saponins, and } \\
\text { alkaloids. }\end{array}$ & $\begin{array}{l}\text { It has diuretic, aphrodisiac, antiurolithic, } \\
\text { immunomodulatory, antidiabetic, absorption } \\
\text { enhancing, hypolipidemic, cardiotonic, central } \\
\text { nervous system, hepatoprotective, anti- } \\
\text { inflammatory, analgesic, antispasmodic, } \\
\text { anticancer, antibacterial, anthelmintic, larvicidal, } \\
\text { and anticariogenic activities. }{ }^{20}\end{array}$ \\
\hline Khadir & $\begin{array}{l}\text { Bark and } \\
\text { Kattha }\end{array}$ & $\begin{array}{l}\text { The major constituents include Catechin, } \\
\text { epicatechin, epigallocatechin, epicatechin } \\
\text { gallate, epigallocatechin gallete rocatechin, } \\
\text { phloroglucin, protocatechuic acid, quercetin, } \\
\text { poriferasterol glucosides, poriferasterol, } \\
\text { acyglucosides, lupenone, lupeol,, kaempferol } \\
\text { and dihydrokaemferol }\end{array}$ & $\begin{array}{l}\text { It has Hepatoprotective, antimicrobial, } \\
\text { cardioprotective, hepatoprotective, Immuno } \\
\text { modulatory, anti-inflammatory, Hypoglycaemic, } \\
\text { antipyretic and antidiarrheal activities. }{ }^{21}\end{array}$ \\
\hline Neem & $\begin{array}{l}\text { Leaf, Flower, } \\
\text { Bark and } \\
\text { Fruit. }\end{array}$ & $\begin{array}{l}\text { The major constituents include quercetin, } \\
\text { azadirachtin, nimbin, nimbinin, nimbidin, } \\
\text { nimbosterol 6-desacetylnimbinene, } \\
\text { nimbandiol, nimbolide, ascorbic acid, } \\
\text { n-hexacosanol and different amino acids. }\end{array}$ & $\begin{array}{l}\text { Neem has Antioxidant, Wound healing, Anti- } \\
\text { inflammatory, Nephroprotective, } \\
\text { Hepatoprotective, antidiabetic, Neuroprotective, } \\
\text { Immuno modulatory activities. }{ }^{22}\end{array}$ \\
\hline Daruharidra & $\begin{array}{l}\text { Root, Fruit, } \\
\text { Steam }\end{array}$ & $\begin{array}{l}\text { It contains mainly berberine, ceryl alcohol, } \\
\text { hentriacontane, sitosterol, palmitic acid, oleic } \\
\text { acid, tannins, flavonoids, phytosterols, } \\
\text { saponins and glycosides. }\end{array}$ & $\begin{array}{l}\text { It has Antitumour, Hypoglycemic, hypolipidemic, } \\
\text { Antidiarrhoeal, Anti-microbial, Anti- } \\
\text { inflammatory activities. }{ }^{23}\end{array}$ \\
\hline Vatsaka & Bark, Seed & $\begin{array}{l}\text { It contains numbers of steroidal alkaloids, } \\
\text { such as conanines, 3- aminoconanines, 20- } \\
\text { aminoconanines, 3-aminopregnans, 20- } \\
\text { diaminopregnanes and their derivatives and } \\
\text { others are holadysenterine, conarrhimine, } \\
\text { isoconessimine, isoconessine etc. }\end{array}$ & $\begin{array}{l}\text { It has mainly Anti-amnesic, Neuroprotective, } \\
\text { Acetylcholinesterase, Antidiabetic, Antiurolithic, } \\
\text { Antibacterial, Anti-inflammatory, Anti-malarial, } \\
\text { Anti-diarrhoeal, Antimutagenic and } \\
\text { Antihypertensive Activities. }{ }^{24}\end{array}$ \\
\hline
\end{tabular}


Table 3: Ayurvedic Properties of Ingredients of Mustadi Kwatha 25,26

\begin{tabular}{|c|c|c|c|c|c|}
\hline Drug name & Rasa & Guna & Veerya & Vipaka & Doshakarma \\
\hline Nagarmotha & $\begin{array}{l}\text { Tikta, Katu, } \\
\text { Kashaya }\end{array}$ & Laghu, Rooksha & Sheeta & Katu & $\begin{array}{l}\text { Kaphapitta Shamaka, Deepana, Pachana, Sangrahi. } \\
\text { Stanyakar, Lekhana, Sothahara, Jworaghna etc. }\end{array}$ \\
\hline Haritaki & $\begin{array}{l}\text { Pancharasa } \\
\text { except } \\
\text { Lavana } \\
\text { Rasa }\end{array}$ & Laghu,Rooksha & Sheeta & Madhur & $\begin{array}{l}\text { Tridoshashamaka, especially Vatashamaka, } \\
\text { Kaphaghna, Srotah-Shodhana, Shothahara, } \\
\text { Vedanasthapana, Chakshushya, Anulomana, Rasayana } \\
\text { etc }\end{array}$ \\
\hline Bibhitaki & $\begin{array}{l}\text { Kasaya } \\
\text { Rasa }\end{array}$ & Rooksha,Laghu & Ushna & Madhur & $\begin{array}{l}\text { Tridoshashamaka, especially Kaphashamaka, } \\
\text { Shothahara, Vedanasthapana, Raktastambhana, } \\
\text { Trishnanigrahana, Chhardinigrahana, Kaphaghna, } \\
\text { Rechana etc. }\end{array}$ \\
\hline Amalaki & $\begin{array}{l}\text { Pancharasa } \\
\text { except } \\
\text { Lavana } \\
\text { Rasa }\end{array}$ & $\begin{array}{l}\text { Guru, Rooksha, } \\
\text { Sheeta }\end{array}$ & Sheeta & Madhur & $\begin{array}{l}\text { Tridoshashamaka, especially Pittashamaka, } \\
\text { Dahaprashamana, Chakshushya, Keshya, Medhya, } \\
\text { Nadibalya, Rochana, Deepana, Anulomana, } \\
\text { Garbhasthapana, Mutrala, Pramehaghna, } \\
\text { Kushthaghna, Jwaraghna, Rasayana etc. }\end{array}$ \\
\hline Haridra & $\begin{array}{l}\text { Tikta, Katu, } \\
\text { Kasaya }\end{array}$ & Rooksha, Laghu & Ushna & Katu & $\begin{array}{l}\text { Kapha Vata Shamaka and Pitta Rechaka, Shothahar, } \\
\text { Vedanasthapan, Varnya, Kusthagna, Vranaropana, } \\
\text { Lekhana, Mutra Viranjniya, Pramehahara etc }\end{array}$ \\
\hline Devdaru & $\begin{array}{l}\text { Tikta, Katu, } \\
\text { Kasaya }\end{array}$ & Rooksha, Laghu & Ushna & Katu & $\begin{array}{l}\text { Kaphavatasamaka, Sothahara, Vedanasthapana, } \\
\text { Kusthagna, Krimigna, Vranasodhana, Vranaropana, } \\
\text { Kaphanisaraka, Pramehagna etc. }\end{array}$ \\
\hline Murva & $\begin{array}{l}\text { Tikta, } \\
\text { Kasaya }\end{array}$ & Guru, Rooksha & Ushna & Katu & $\begin{array}{l}\text { Tridoshahara, Amapachana, Anulomana, } \\
\text { Shulaprasamana, Krimighna, Raktasodhaka, } \\
\text { Pramehagna, Jworaghna, Kusthagna etc. }\end{array}$ \\
\hline Indravaruni & Tikta & $\begin{array}{l}\text { Laghu, Rooksha, } \\
\text { Teekshna }\end{array}$ & Ushna & Katu & $\begin{array}{l}\text { Kaphapittahara, Rechaka, Vishagna, Vamak, } \\
\text { Krimighna, Vranasodhana, Raktasodhaka, } \\
\text { Kaphanisaraka, Pramehagna, Jwaragna etc. }\end{array}$ \\
\hline Lodhra & Kasaya & Laghu, Rooksha & Sheeta & Katu & $\begin{array}{l}\text { Kaphapittasamaka, Kusthagna, Sothahara, } \\
\text { Chakshushya, Raktastambhana, Varnaropana, } \\
\text { Shonitasthapana, Stambhana, Mridurechana, } \\
\text { Raktashosaka, Kaphaghna, Jwaraghna etc. }\end{array}$ \\
\hline Gokshura & Madhura & Guru Snigdha & Sheeta & Madhura & $\begin{array}{l}\text { Vatapitta Shamaka, Vedanasthapana, Anulomana, } \\
\text { Raktapitta Shamaka, Ashmarinashana, Mutral, Valya } \\
\text { etc. }\end{array}$ \\
\hline Khadir & $\begin{array}{l}\text { Tikta, } \\
\text { Kasaya }\end{array}$ & Laghu, Ruksha & Sheeta & Katu & $\begin{array}{l}\text { Kusthagna, Kaphapitta Shamaka, Sthmbhana, } \\
\text { Krimighna, Raktaprasadak, Jworagna, } \\
\text { Medadhatusoshana, Sothagna etc. }\end{array}$ \\
\hline Neem & $\begin{array}{l}\text { Tikta, } \\
\text { Kasaya }\end{array}$ & Laghu & Sheeta & Katu & $\begin{array}{l}\text { Kaphapitta Shamaka, Varnasodhana, Kusthagna, } \\
\text { Dahaprashaman, Kandughna, Vedanasthapan, } \\
\text { Krimighna, Yekritautejaka, Rkatasodhaka, } \\
\text { Pramehahara, Jwaraghna etc. }\end{array}$ \\
\hline Amaltas & Madhura & $\begin{array}{l}\text { Guru, Mirdu, } \\
\text { Snigdha }\end{array}$ & Sheeta & Madhura & $\begin{array}{l}\text { Vatapitta Shamaka, Kaphapitta Samsodhaka, } \\
\text { Kusthagna, Vedanasthapana, Anulomana, } \\
\text { Mriduvirechaka, Kaphanisharaka, Raktasodhaka, } \\
\text { Hridya, Mutrajanan Aamasodhaka etc. }\end{array}$ \\
\hline Daruharidra & $\begin{array}{l}\text { Tikta, } \\
\text { Kasaya }\end{array}$ & Laghu, Rukshya & Ushna & Katu & $\begin{array}{l}\text { Kaphapitta Shamaka, Sothahara, Vedanasthapana, } \\
\text { Varnasodhana, Varnaropana, Yekritutejaka, Deepan, } \\
\text { Raktasthamvaka, Jwaragna etc. }\end{array}$ \\
\hline Patha & Tikta & Laghu Tikshna & Ushna & Katu & $\begin{array}{l}\text { Tridoshasamak, Mainly Kaphapitta Shamaka. } \\
\text { Varnaropana, Vishagna, Kusthagna, Krimigna, } \\
\text { Raktashodhaka, Deepan, Pachana, Sothahara, } \\
\text { Stanyashodhana etc }\end{array}$ \\
\hline Vatsaka & $\begin{array}{l}\text { Tikta, } \\
\text { Kasaya }\end{array}$ & Laghu Ruksha & Sheeta & Katu & $\begin{array}{l}\text { Kaphapittashamaka, VArnaropana, Sthambhana, } \\
\text { Arshoghna, Sangrahik, Raktasthambaka, Jworagna, } \\
\text { Dhatusoshana etc. }\end{array}$ \\
\hline
\end{tabular}




\section{CLINICAL STUDIES}

\section{Anti-hyperlipidemic effect}

Study by Nadkarni M. A. et al. have shown that administration of Mustadi Ghanavati for hyperlipidemia patient decreased serum cholesterol by $22.4 \%$, serum triglycerides by $19.6 \%$, serum LDL by $18.2 \%$, serum VLDL by $4.2 \%$ and increased serum HDL by $5.6 \%$. 27 In another clinical study $40 \mathrm{ml}$ of Mustadi Kwatha was administered to dyslipidaemia patient for 90 days. It shows that there were statistically significant improvement in biochemical parameters, such as serum cholesterol, Sr. triglycerides, Sr. LDL, and Sr. VLDL. Researcher also highlighted that there was statistically highly significant improvement in subjective parameters like Anga Gaurava, Kshudraswasa, Angmarda, Javoparodha and statistically significant result in Aruchi, Atisweda, Karpaddaha, Nidraatiyoga. 28

\section{Anti- diabetic effect}

In one study it was reported that there was a statistically significant reducing effect $(p<0.001)$ of Mustadi Kwatha Ghanavati $500 \mathrm{mg}$ interventions on fasting blood glucose, post prandial blood glucose, urine glucose, total-cholesterol systolic blood pressure and diastolic blood pressure levels than placebo treatment. ${ }^{29}$ In another study by Jayanta Kumar Sarma et al. have shown that the administration of Mustadi
Kwatha provides significant relief in objective parameters like Fasting blood Sugar, Post Prandial blood sugar, Glycated haemoglobin (HbA1C) and in subjective parameter like Prabhoota and Avila Mootrata after 90 days of treatment. ${ }^{30}$

\section{CONCLUSION}

Mustadi Kwatha is a well-known Ayurvedic formulation which has been used since vedic period for the treatment of different metabolic disorders such as diabetes mellitus, hyperlipidemia, obesity etc. Trying to cure lifestyle related chronic disease with pharmaceutical medicine is like, "trying to dry out a flooded room without turning off the tap." So it's a time to search and explore traditional medicines which have the ability to treat lifestyle related diseases. This study contributes in exploring different pharmacodynamics and clinical expect of Mustadi Kwatha. After reviewing different classical Ayurvedic text books and scientific research papers, we can suggest that Ayurvedic polyherbal formulation Mustadi Kwatha can be used in the treatment of lifestyle related diseases.

\section{CONFLICT OF INTEREST}

There are no any conflicts of interest.

FUNDING: None.

\section{REFERENCES}

1. Agnivesa, Caraka Samhita, Sutra Sthana, Verse no. 23/12-13. Elaborated by Caraka and Drdhabala, Commentary by Sri Cakrapanidatta, Edited by Vaidya Yadavji Trikamji Acharya, Prologued by Prof. R.H. Singh; Chaukhamba Surbharati Prakashana, Varanasi, Ed. 2017 ; Pg.122.

2. Kaviraj Govind Das Sen, Bhaisajya Ratnavali, Verse 37/18, Edited with Siddhiprada Hindi Commentary by Prof. Siddhi Nandan Mishra,Chaukhamba Surbharati Prakashan, Varnasi,Ed.2017;Pg.697

3. Pandita Sharangadharacharya Sharangadhara Samhita, Hindi commentary by Dr. Brahmananda Tripathi. Choukhambha Surbharti Prakashan Vanarasi; Ed. 2017 Pg. 90.

4. Agnivesa, Caraka Samhita, Sutra Sthana, Verse no. 23/12-13. Elaborated by Caraka and Drdhabala, Commentary by Sri Cakrapanidatta, Edited by Vaidya Yadavji Trikamji Acharya, Prologued by Prof. R.H. Singh; Chaukhamba Surbharati Prakashana, Varanasi, Ed. 2017 ; Pg.122.

5 Kaviraj Govind Das Sen, Bhaisajya Ratnavali, Verse 37/18, Edited with Siddhiprada Hindi Commentary by Prof. Siddhi Nandan Mishra,Chaukhamba Surbharati Prakashan, Varnasi, Ed.2017;Pg.697

6. Dr. J.L.N. Sastry Illustrated Madanpala Nighantu, Chaukhambha Orientalia, Varanasi, Edition 2010.

7. Prof. P. V. Sharma Dravyaguna Vijnana Vol II, Chaukhambha Bharati Academy Gokul Bhawan, Gopal Mandir Lane, Varanasi, India; Ed.2009. 8. Kilani S., Sghaier M.B., Limem I., Bouhlel I., Boubaker J., Bhouri W., Ghedira K., In vitro evaluation of antibacterial, antioxidant, cytotoxic and apoptotic activities of the tubers infusion and extracts of Cyperus rotundus. Bioresource technology, 2008; 99(18):9004-9008.

9. Imam H., Sofi G., Seikh A., Lone A. The incredible benefits of Nagarmotha (Cyperus rotundus). International Journal of Nutrition, Pharmacology, Neurological Diseases, 2014; 4(1):23.

10. Meher S. K., Panda P., Das B., Bhuyan G. C., \& Rath K.K., Pharmacological Profile of Terminalia chebula Retz. and Willd.(Haritaki) in Ayurveda with Evidences. Research Journal of Pharmacology and Pharmacodynamics, 2018; 10(3):115-124.

11. Gupta A., Kumar R., Kumar S., Pandey A.K., Pharmacological aspects of Terminalia bellirica. Molecular Biology and Pharmacognosy of Beneficial Plants (Eds. AA Mahdi, M. Abid, MMAA Khan, MI Ansari, RK Maheshwari), Lenin Media Private Limited: Delhi, India, 2017 ; 52-64.

12. Bhat P. M., Umale H., \& Lahankar M. Amalaki: A review on functional and pharmacological properties. Journal of Pharmacognosy and Phytochemistry, 8(3), 4378-4382.

13. Akram M., Shahab-Uddin A. A., Usmanghani K. H. A. N., Hannan A. B. D. U. L., Mohiuddin E., \& Asif, M. Curcuma longa and curcumin: a review article. Rom J Biol Plant Biol, 2010; 55(2):65-70.

14. Patel S. J., Lambole V., Shah P., \& Shah D.P. Pharmacological Activities Of Cedrus Deodara: An Overview. Pharma Science Monitor, 2013; 3(3). 15. Wang P., Yang J., Zhu Z., \& Zhang X. Marsdenia tenacissima: a review of traditional uses, Phytochemistry and pharmacology. The American journal of Chinese medicine, 2018; 46(07):1449-1480.

16. Pravin B., Tushar D., Vijay P., Kishanchnad K. Review on Citrullus colocynthis. Int. J. Res. Pharm. Chem, 2013; 3(1):46-53. 
17. Singh P., Singh R., Gupta L. ., \& Kumar N. Lodhra: A single remedy for different ailments. Int J harm Biological archives, 2005; 6(1):1-7.

18. Mondal M., Hossain M. M., Rahman M. A., Mubarak M.S., PK M.M.U., Islam M. S., \& Das, N. Cassia fistula Linn: a review of phytochemical and pharmacological studies. International Journal of Pharmaceutical Sciences and Research, 2014; 5(7):2125-30.

19. Kudale R. R., Kulkarni D. V., Pawar R.S. Pharmacological Profile of Patha (Cissampelos Pareira Linn.): A Review. 2019.

20. Chhatre S., Nesari T., Somani G., Kanchan D., Sathaye S. Phytopharmacological overview of Tribulus terrestris. Pharmacognosy reviews, 2014; 8(15):45.

21. Lakshmi T., Geetha R.V., Anitha R. Acacia Catechu Willd: A Pharmacological Review. International Journal of Current Research and Review.2011; 3(5):101-111.

22. Rahmani A., Almatroudi A., Alrumaihi F., \& Khan A. Pharmacological and therapeutic potential of neem (Azadirachta indica). Pharmacognosy Reviews, 2018; 12(24).

23. Tamilselvi S., Balasubramani S.P., Venkatasubramanian P.A.D.M.A., Vasanthi N.S., A review on the pharmacognosy and pharmacology of the herbals traded as 'daruharidra'. Int J Pharm Bio Sci, 2014; 5(1):556-70.

24. Sinha S., Sharma A., Reddy P. H., Rathi B., Prasad N.V.S.R.K., \& Vashishtha A. Evaluation of phytochemical and pharmacological aspects of Holarrhena antidysenterica (Wall.): A comprehensive review. Journal of pharmacy research, 2013; 6(4):488-492.

25. Dr. J.L.N. Sastry Illustrated Madanpala Nighantu, Chaukhambha Orientalia, Varanasi, Ed. 2010.

26. Prof. P. V. Sharma Dravyaguna Vijnana Vol II, Chaukhambha Bharati Academy Gokul Bhawan, Gopal Mandir Lane, Varanasi, India; Ed.2009.

27. Nadkarni M. A., Vyas S. N., Baghel M. S., \& Ravishankar B. Randomized placebo-controlled trial of Mustadi Ghanavati in hyperlipidemia. Ayu, 2010; 31(3):287.

28. Pandey J, Singh OP, Shukla S, Sujatha N; A Clinical Study to Evaluate the Efficacy of Mustadi Kwath in Dyslipidemia vis-à-vis Medodusti; Int J Ayu Pharm Chem 2018; 9(1):195-201.

29. Kushwaha, V., Kar, A. Clinical study on Ayurvedic herbal drug (Mustadi Kwatha Ghanavti) therapy in patients with Type 2 Diabetes, 2017.

30. Sarma JK, Vineeta V. Deshmukh. Comparative study to evaluate the effect of an Indigenous Compound Mustadi Kwatha in the treatment of Madhumeha w.s.r Diabetes Mellitus (NIDDM). The antiseptic, 2020; 117. 